\title{
Introdução ao XBRL - nova linguagem para a divulgação de informações empresariais pela internet
}

\section{Edson Riccio}

Professor dos cursos de graduação e de pós-graduação da FEA/USP.

E-mail: elriccio@usp.br

\section{Marici Sakata}

Doutoranda e mestra em ciências da comunicação pela Escola de Comunicações e Artes (ECA).

E-mail: mcsakata@usp.br

\section{Orandi Moreira}

Pesquisador do TECSI - Laboratório de Tecnologia e Sistemas de Informação. Mestre em contabilidade e controladoria pela Faculdade de Economia, Administração e Contabilidade da USP.

E-mail: orandi@usp.br

\section{Luc Quoniam}

Professeur de Université du SUD Toulon-Var, França e professor visitante da FEA USP. E-mail: quoniam@usp.br

\section{Resumo}

O trabalho tem como objetivo analisar as principais características de divulgação de informações financeiras pela Internet, por meio da linguagem eletrônica XBRL - eXtensible Business Reporting Language. Foram estudados a sua trajetória, estado da arte, funcionalidades, principais locais de desenvolvimento, grupos de pesquisa, institutos envolvidos, eventos relacionados, taxonomia de sua estrutura, tendo como base a bibliografia sobre o tema.

\section{Palavras-chave}

Difusão de informações. Governança. Internet. Relatório financeiro. XBRL. XML.

\section{Introduction to XBRL - the new language for corporate disclosure throughout the internet}

\begin{abstract}
The objective of this paper is to analyze the main characteristics of dissemination of funding information through Internet, by means of the XBRL electronic language /ExTensible Business Reporting Language. The main subjects studied are XBRL trajectory, state-of-art, functionalities, principal places of development, research groups, institutes involved, events related, taxonomy of its structure, having as a basis the bibliography about the theme.
\end{abstract}

\section{Keywords}

Corporate governance. Disclosure. Financial statement. Internet. XBRL. XML.

\section{INTRODUÇÃO}

A globalização, a nova economia e a sociedade da informação estão presentes no dia-a-dia, independentemente das atividades a que se dediquem. Os países e as pessoas estão cada vez mais interligados. Antes, pelos meios tradicionais de comunicação, e agora, principalmente por meio da Internet. As empresas abertas divulgam suas informações financeiras visando a atender às legislações específicas e às entidades reguladoras dos mercados em que atuam. As empresas também precisam passar uma nova imagem à sociedade, com a qual têm a intenção de se mostrarem modernas, abertas e transparentes. Para tanto, necessitam meios capazes de transmitir informações financeiras de forma mais ágil e dinâmica do que aquela observada até o momento.

Este trabalho tem como objetivo principal analisar as principais características desta forma de divulgação de informações empresariais, a evolução do XBRL e o estado-da-arte da linguagem.

\section{METODOLOGIA}

Para que se possa saber o grau de desenvolvimento notado em um país ou em um segmento de mercado, partindo dos conceitos, é necessário buscar meios de quantificar a utilização ou o envolvimento de uma nação ou de um segmento de mercado no objeto em estudo.

Para essa etapa inicial de estudos, a técnica utilizada foi a revisão bibliográfica e documental. Os resultados obtidos permitiram que dados qualitativos fossem levantados, gerando bases para uma posterior análise das mudanças. Estudou-se o surgimento e a evolução das chamadas linguagens de marcação, a evolução da linguagem XML e o desdobramento de sua vertente para demonstrações financeiras como o XBRL, buscando-se suas características, seus módulos principais, e estudou-se o que vem a ser a chamada taxonomia necessária para se utilizar a linguagem.

Fez-se então uma passagem literária por vários países do mundo, buscando-se saber como estão sendo desenvolvidos os estudos desta linguagem, qual a forma de organização das entidades envolvidas com estes 
estudos, qual o estágio de desenvolvimento já detectado no objeto em estudo e comparativamente como estão estes estudos no Brasil.

\section{A EVOLUÇÃO DAS LINGUAGENS DE MARCAÇÃO (MARKUP LANGUAGE)}

A origem das linguagens de marcação data de 1960, quando a IBM, avaliando a comunicação então existente entre diversos sistemas, constatou que diferentes tipos deles não conversavam entre si. Seu departamento de engenharia propôs, como solução, a primeira linguagem de marcação, que chamou GML, Generalized Markup Language. Conceitualmente, linguagem de marcação não é simplesmente uma linguagem, mas sim uma chamada metalinguagem. Sua proposta é descrever um método para descrição de dados, de forma a estabelecer uma série de regras e procedimentos que permitam identificar como funciona um conjunto de informações, ou seja, um protocolo de normas (ALIANA, 2004, p.4). O termo Markup vem a ser as marcas, ou labels, que identificam onde e como a informação deverá aparecer, tornando-a assim uma linguagem que permite construir uma linguagem.

Marcação é a informação incluída em um documento para melhorar seu significado, por identificar as partes e como elas se relacionam, e "inguagem de marcação é um conjunto de símbolos que pode ser colocado no texto de um documento para demarcar e rotular as partes desse documento (RAY, 2001, p. 3).

A ISO, International Organization for Standardization, reconheceu em 1986 a primeira linguagem de marcação, chamada SGML - Standard Generalized Markup Language -, a qual recebeu o ISO 8879 (ISO, 2004). Esta linguagem, sendo de marcação, não especifica uma formatação particular, mas sim as regras para marcar os elementos, de maneira que essas marcas possam ser interpretadas para formatar elementos em diferentes formas. Ela é ainda bastante utilizada quando se deseja manipular grandes elementos, que podem estar sujeitos a alterações freqüentes e necessitar serem apresentados em diferentes formatos. Mas, com o tempo, demonstrou ser demasiadamente complexa e também requerer grandes áreas de trabalho, o que dificultou sua adoção em larga escala, principalmente em computadores pessoais. Mas serviu de base para as próximas markup language que viriam a ser desenvolvidas (WEBOPEDIA, 2004).

O desenvolvimento continuou e, em 1990, Tim BernersLee, físico suíço, liderou o desenvolvimento de uma linguagem que chamou HTML - Hypertext Markup Language - e deu nome a seu sistema de WWW, World
Wide Web -, que, sabe-se, foi aquele em grande parte responsável pela "explosão" do fenômeno Internet (HOFFMAN; STRAND, 2001, p.35). Este chamado hipertexto é na verdade um documento que possibilita incluir em seu conteúdo ligações com outras partes desse mesmo documento ou com documentos diferentes. Essas ligações normalmente são indicadas por meio de uma imagem ou texto em uma cor diferente ou sublinhado. Quando se clica na ligação, o usuário é levado até o texto ligado (PICONEZ, 2003, p.4).

\section{A linguagem XML}

Quando parecia que a HTML viria resolver o problema de complexidade até então verificado com a SGML, constatou-se que sua simplicidade, por sua vez, acabava sendo fator de muitas limitações, pois era muito estática e permitia apenas que a informação fosse apresentada, não possibilitando outras formas de interação. Segundo Aliana (2004, p.4),

um arquivo em formato HTML permite mostrar os estados financeiros de uma companhia, mas impede o usuário de saber como foram construídas essas cifras.

A solução foi dada em 1998, com o W3C, World Wide Web Consortium, um consórcio internacional que desenvolve tecnologias para a web, que disponibilizou formalmente a linguagem XML - eXtensible Markup Language -, que também é compatível com a linguagem SGML, mas considerada bem menos complicada, e possibilita incorporar códigos de formatação com grande variedade de programas de computador.

Hoffman e Strand (2001, p.35) defendem que a linguagem XML é bem fundamentada teoricamente, pode ser vista e editada com qualquer editor de texto e pode ser lida e entendida tanto pelos computadores quanto por humanos. Já para Light (1999, p.47), receber XML, em vez de HTML, torna-o muito mais auto-suficiente, pois os processadores XML genéricos lhes permitirão carregá-la usando uma tabela virtual de conteúdo gerada pelo próprio documento e assim os clientes não precisarão retornar ao servidor simplesmente para ver a informação de uma forma diferente. Assim, se você quiser uma visão diferente da informação, somente terá de carregar uma folha de estilo diferente.

quadro 1, a seguir, apresenta um documento-texto (parcial).

Já o quadro 2 apresenta um possível correspondente documento XML das informações. 
QUADRO 1

Documento-texto

\begin{tabular}{|c|c|c|c|}
\hline \multirow[b]{2}{*}{ IN MILLIONS OF DOLLARS } & \multicolumn{3}{|c|}{ December } \\
\hline & 1999 & & 1998 \\
\hline Assets & & & \\
\hline Cash and cash equivalents & $\$ 957$ & . & $\$ 550$ \\
\hline Accoounts receivable & 4,337 & & 3,417 \\
\hline Inventories and contracts in progress & 3,504 & & 3,191 \\
\hline Future income tax benefits & 1,563 & & 1,222 \\
\hline Other current assets & 266 & - & 161 \\
\hline Net investment in discontinued operation & & & $\ldots 1,287$ \\
\hline
\end{tabular}

Fonte: Hoffman e Strand (2001, p. 37).

\section{QUADRO 2}

Documento XML

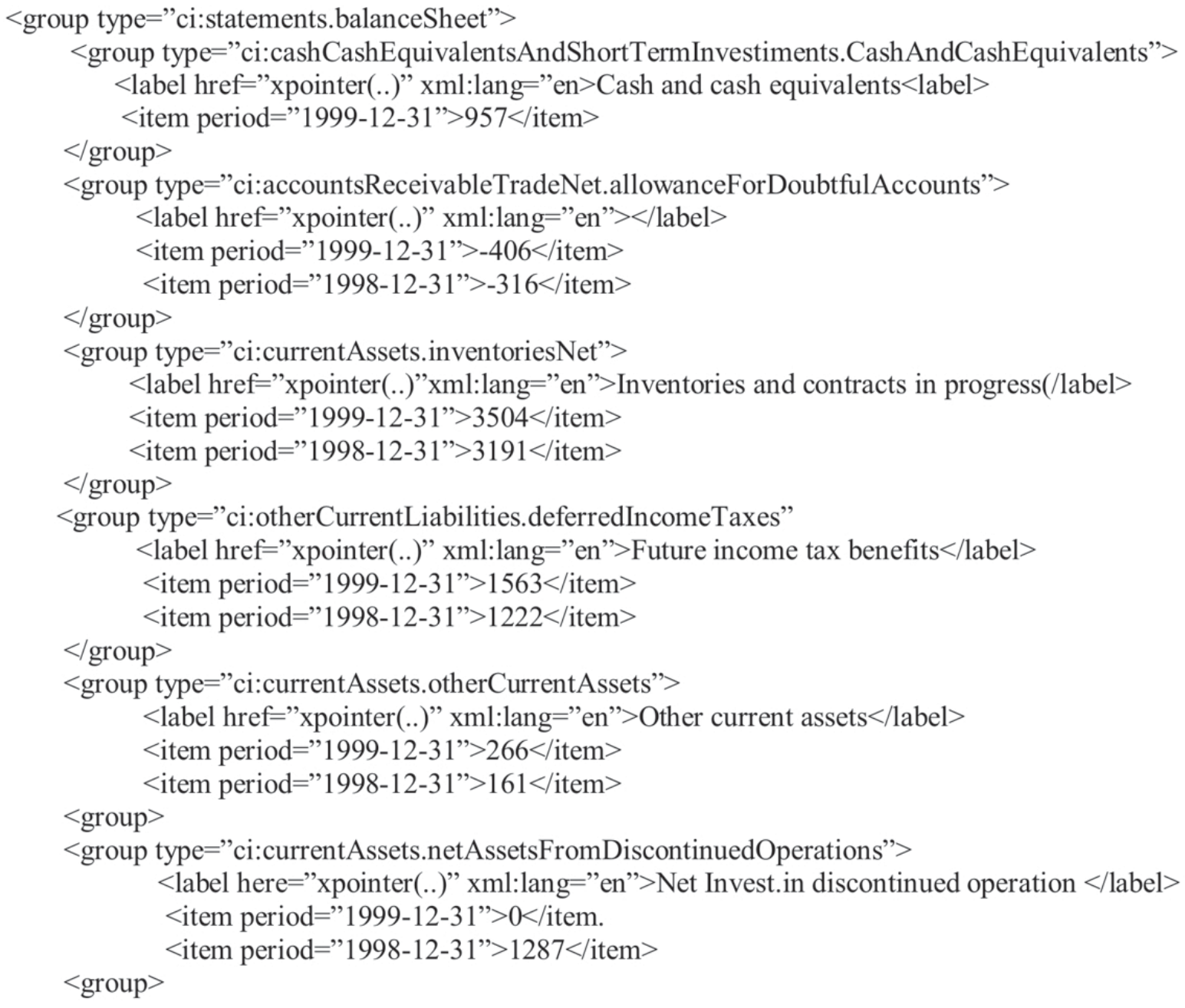

Fonte: Hoffman e Strand (2001, p. 38). 
O que se observa é que cada informação é marcada (markup) quando se usa o XML. Esta é a solução encontrada para que o computador possa entender a informação. Posteriormente, estando no formato XML podem ser usadas folhas de estilo (style sheet) para transformar a informação para o formato que se deseje, como formato texto para impressão, HTML, Word, Planilha, Banco de Dados, ou outros. Para realizar essa transformação, já foi desenvolvida a ferramenta XSLT eXtensible Stylesheet Language Transformations -, a qual facilita fazer a transformação para o formato requerido.

Para Aliana (2004, p. 5)

(...) o XML se configura assim como uma linguagem universal, tanto para formatar como para apresentar a informação. Uma vez que os dados estão codificados ou identificados com etiquetas, estes podem ser utilizados de formas diferentes. Através de uma série de regras, guias e convenções, desenham o formato dos textos e dos dados de modo que pode facilmente ser lido e gerado por um ordenador, de forma não ambígua e evitando falhas comuns como falta de exportabilidade ou dependência específica de uma plataforma.

Hoffman e Strand (2001, p. 42) afirmam ainda que XML é uma linguagem para construir linguagens e, dessa maneira, não poderia uma única linguagem servir para todos os tipos de necessidades. Uma extensa gama de aplicações para a XML vem surgindo ao longo do tempo, e com isso essa linguagem está sendo utilizada em diversos campos, como o da matemática e da física, bem como tem sido aplicada a atividades de negócios, como no caso do comércio eletrônico, entre outros. Assim, cada grupo desenvolve a linguagem XML voltada para suas necessidades específicas. $O$ quadro 3 , a seguir, apresenta algumas dessas variantes específicas já existentes.

\section{A linguagem XBRL}

A linguagem XBRL é uma das variantes da XML, com o propósito de se tornar a linguagem-padrão para divulgação de demonstrativos financeiros, que está sendo desenvolvida pelo consórcio XBRL International.

O início do desenvolvimento da XBRL data de abril de 1998, com Charles Hoffman, um CPA, Certified Public Accountant, da empresa Knight Vale and Gregory, em Tacoma, Washington, que passou a pesquisar o uso da linguagem XML para divulgar informações financeiras para divulgação eletrônica (TRUMAN, 2004). Em julho
QUADRO 3

Exemplos de linguagens XML específicas

CML, Chemical Markup Language (CML ${ }^{\mathrm{TM}}$, 2005)

EbXML, Electronic business information (ebXML, 2005)

FinXML, the digital language for capital markets (FinXML ${ }^{\mathrm{TM}}$, 2005)

FpXML, the XML Standard for Swaps, Derivatives and Structured Products (FpML TM, 2005)

HR-XML, automation of human resources-related data exchanges (HR-XML CONSORTIUM, 2005)

NewsML, News Markup Language for global news exchange (XMLNEWS.ORG, 2005)

XBRL, eXtensible Business Reporting Language (XBRL INTERNATIONAL, 2004)

de 1998, ele levou suas idéias ao AICPA, American Institute of Certified Public Accountants, que se interessou pela idéia e criou um projeto para desenvolvimento de um protótipo da linguagem, o qual já foi apresentado em 15 de janeiro de 1999 (XBRL HISTORY, 2004).

O AICPA então se convenceu da utilidade da linguagem e, em junho de 1999, deu início a um projeto que teve o nome inicial de XFRML, eXtensible Financial Reporting Markup Language. Em agosto daquele ano, mais 11 companhias também se juntaram ao projeto e formaram o comitê de desenvolvimento da linguagem, sob o comando do AICPA. Eram elas Arthur Andersen, Deloitte \& Touche, e-content Company, Ernest \& Young, Edgar Online, FRx Software Corporation, Great Plains, KPMG, Microsoft Corporation, PricewaterhouseCoopers e a The Woodburn Group. Em outubro daquele ano, já acontecia o primeiro evento público sobre o assunto, na cidade de Nova Iorque, sendo que neste encontro já se definiu que seria criada a primeira taxonomia dirigida para divulgação financeira dos setores comercial e industrial dos Estados Unidos, uma vez que estas representavam cerca de $80 \%$ das companhias norte-americanas (XBRL HISTORY, 2004).

O consórcio teve seu nome mudado oficialmente para XBRL em abril de 2000, sendo que, em julho daquele mesmo ano, anunciam-se não só a conclusão da taxonomia para companhias comerciais e industriais dos Estados Unidos, mas também a internacionalização do consórcio, visando à rápida expansão da linguagem. 
Em fevereiro de 2001, realizou-se a primeira XBRL International Conference em Londres, contando com representantes de 10 países. Nesse evento, a IASB, International Accounting Standards Board, já apresentou, para ser apreciada, uma versão da taxonomia IAS, International Accounting Standards. Também o ICAEW, The Institute of Chartered Accountants in England and Wales, anunciou a formação de um grupo de trabalho para desenvolver a versão UK, United Kingdown, da taxonomia XBRL.

Dessa maneira, fica claro que o XBRL não surgiu por acaso nem foi uma descoberta, mas, como acontece em todas as áreas da ciência, é fruto de uma evolução de conhecimentos. Para Debreceny e Gray (2004), o XBRL foi desenhado para suportar todos os formatos de relatórios financeiros e de negócios e, sendo baseado na linguagem XML, aumenta a velocidade do envio de informações eletrônicas.

$\mathrm{Na}$ liderança de seus estudos e pesquisas nos Estados Unidos e no mundo, o AICPA está apostando fortemente na linguagem e encabeça o consórcio internacional XBRL. Zarowin e Harding (2000, p.1), dois de seus pesquisadores, afirmam que:

XBRL, Extensible Business Reporting Language, em breve será a língua padrão para todos os relatórios financeiros, desde a distribuição de informações financeiras a bancos e investidores para cumprir as regras da SEC ou carregar informações financeiras em um site da web. $\mathrm{O}$ desenvolvimento certamente irá revolucionar a maneira como a informação é disponibilizada, usada e calculada.

Segundo um artigo publicado no The CPA Journal, o AICPA anunciou naqueles dias sua lista das top 10 technologies que mais iriam afetar a profissão contábil em 2004, sendo que, entre essas 10, estava a tecnologia de troca de informações de negócios, via Internet, tendo citado especificamente a linguagem XBRL (TOP, 2004).

\section{A distinção HTML, XML e XBRL}

Apesar de os termos HTML, XML e XBRL estarem fortemente relacionados, precisa-se fazer a distinção clara entre cada um deles.

HTML é um sistema de marcação de documento, de maneira que ele possa ser publicado na "rede mundial www". Os documentos HTML contêm referências gráficas e rótulos (tag) que se preocupam em descrever como o documento irá aparecer na Internet. Como exemplo, pode-se dizer que ele descreve qual a fonte, cor, tamanho e outras características necessárias, quando se vai enviar um texto para a tela. Porém, ele contém somente dados, e não informações, porque na verdade tudo precisa lhe ser informado para que ele possa publicar a informação.

A XML, por sua vez, também usa "tag", entretanto não limita um predefinido número desses "tags", mas sim permite uma rede desses labels predefinida (taxonomia) e as devidas relações entre elas. A linguagem XML é mantida pelo Consórcio W3C, na qual os dados são independentes e podem ser encaminhados a qualquer dispositivo, como computador, telefone celular e outros, e permite a seus desenvolvedores oferecer os dados de maneira uniforme e consistente.

E, finalmente, o XBRL é baseado na linguagem XML, tendo sido desenvolvido especificamente para a emissão de relatórios financeiros, sendo assim um complemento daquela linguagem, permitindo que os usuários identifiquem de maneira única os itens que são disponibilizados em seus demonstrativos. O XBRL é um software livre e aberto desenvolvido pelo consórcio XBRL International.

\section{Componentes da XBRL}

A XBRL possui dois componentes fundamentais: a taxonomia e o instance document. Eles serão usados simultaneamente, para extrair suas informações no formato padronizado. Após isso, um terceiro elemento, o style sheet, pode ser usado como complemento no momento da conversão da saída para o formato desejado.

Hoffman e Strand (2001, p.68) entendem que a taxomonia, definindo os fatos financeiros a serem descritos nos relatórios, constitui-se do vocabulário ou dicionário de termos a serem usados no instance document, na forma como eles se relacionam entre si, sendo que uma ou mais taxonomias podem ser usadas em um único instance document. No instance document é que será informado à aplicação o valor que esses elementos terão naquele momento. Da leitura e interpretação desses dois elementos, a aplicação XBRL gerará as informações sobre os relatórios financeiros, no formato de arquivo XML.

Estas informações poderão então ser baixadas para outros programas, que farão processamentos posteriores, ou então poderão ser formatadas para visualização, aplicando o terceiro componente, style sheet, que permitirá transformar as informações para qualquer formato 
desejado, como outro arquivo XML, ou um arquivo PDF, Portable Document Format, arquivo em formato HTML, arquivos impressos ou qualquer outro tipo de saída desejada.

\section{A Taxonomia XBRL}

Os grandes estudos e discussões atuais da XBRL estão focados na definição das taxonomias específicas para seus usuários. É na taxonomia, por meio de um vocabulário controlado, que poderão ser construídos documentos para a área financeira. Essa especificação dos dados, que é a explicação técnica do que vem a ser o XBRL e de como funciona (metalinguagem) forma a base da linguagem e viabiliza a função principal da linguagem. Esta função é facilitar a divulgação de ampla gama de conteúdos contábil-financeiros, apesar da complexidade das informações e regras que esses devem seguir, de maneira que possam ser facilmente compartilhados, compreendidos e manuseados pelos usuários.

A taxonomia define os termos que serão utilizados nos demonstrativos, assim como os tipos de dados que serão abrigados por cada um destes termos, que serão dados pelas especificações de cada item, e ainda as relações entre cada um destes termos, que resultarão em um relatório final. De acordo com a Encyclopaedia Britannica, o termo taxonomia, na biologia, é a classificação de um organismo em hierarquias de agrupamento, do geral para o particular, que reflete as relações evolucionais e morfológicas. Assim, de uma forma geral, entende-se por taxonomia o estudo de princípios gerais de classificação científica (MERRIAN-WEBSTER, 2004). Aplicado ao XBRL, o esquema básico, que define as estruturas dessa taxonomia, pode ser visualizado na figura 1 .

Assim, a taxonomia da XBRL pode ser entendida como um dicionário para fornecer definições-padrão para as informações dos relatórios financeiros, construído a partir de sua estrutura e hierarquias. Nele estariam contidas as descrições dos dados numéricos e textuais a serem reportados em cada caso específico e preparados de acordo com tal especificação. $O$ cuidado que está sendo tomado na construção dessa taxonomia é decorrência da complexidade que existe quando se pensa em relatórios financeiros oriundos de diferentes tipos de organização, devendo seguir diferentes padrões, regras e regulamentos de diversos países, e que contêm os mais variados tipos de informações. É então a taxonomia que irá permitir a característica de extensibilidade, ou expansibilidade ou customização, do XBRL. Para Hoffman e Strand (2001, p.57),

o XBRL precisa contemplar as necessidades de todos os participantes da cadeia de usuários de relatórios financeiros. Isto inclui diferentes organizações em diferentes países e jurisdições contábeis, todos com diferentes pontos de vista do mundo. Desta maneira, a extensibilidade é uma característica crítica do XBRL.

Para satisfazer às necessidades de cada jurisdição contábil, cada setor da indústria, cada organização individual, é que o XBRL foi concebido buscando ser extensível, solução encontrada nas chamadas taxonomias. Outra característica encontrada na XBRL é a flexibilidade que proporciona, pois foi concebida possibilitando criar pares nome-valor, de maneira a fornecer informações fundamentais sobre eles, como atributos, tipos de dados, formatos e outros. E isto pode ser explorado visando a adaptar a linguagem às diferentes características das entidades envolvidas. 
Para Silva e Teixeira (2004, p.6), a taxonomia é a biblioteca dos termos financeiros usados na preparação dos relatórios, e sua função é definir o conjunto de elementos, com seus atributos, e os relacionamentos que ocorrem entre si. Na especificação de uma taxonomia, vários elementos são relatados visando a descrever como serão aceitos os dados informados e como serão disponibilizadas as informações. $\mathrm{O}$ quadro 4 contém as informações requeridas para criação de uma taxonomia XBRL.

No quadro 5, é apresentado um exemplo ilustrativo do formato característico de uma taxonomia.

\section{QUADRO 4}

Lista de informações necessárias para criação de taxonomia XBRL

Name: nome do elemento.

Type: tipo de dado que é o elemento descrito.

Documentation: uma descrição do fato financeiro.

To: para onde o dado está direcionado.

From: de onde provém o dado.

Weight: indica relação matemática do elemento filho para com o pai, que pode ser de adição (1), subtração (-1) ou nenhum (0).

Order: ordem física de apresentação do elemento.

Label: rótulo do elemento, o qual pode ser diferente para os diferentes idiomas utilizados.

Fonte: Silva e Sulaiman (2003, p.7).

\section{QUADRO 5}

\section{Taxonomia em XML}

$<$ ?xml version $=" 1.0 "$ encoding $=$ "utf-8"? $>$

$<!$-Created:7/28/2000 5:11:16 PM $\rightarrow$

$<!$ - - targetNamespace names what we are defining $->$

<schema xmlns=http://www.w3.org/1999/XMLSchema"xmlns:http://www.w3.org/1999/xhtml"xmlns:xbrl=http://www.xbrl.org/core/ 2000-07-31/metamodeltargetNamespace $=$ http://www.xbrl.org.us/gaap/ci/2000-07-31>

$<$ import namespace $=$ http://www.xbrl.org/core/2000-07-31/metamodel schemaLocation $=$ http://www.xbrl.org/core/2000-07-31/ xbrl-meta-2000-07-31.xsd/>

$<$ element name $=$ "statements"type $=$ "string" $>$

$<$ annotation $>$

$<$ appinfo $>$

$<$ xbrl:label xml:lang="en" $>$ Statements $<\mid$ xbrl:label $>$

$<$ appinfo $>$

$<$ annotation $>$

$</$ element $>$

$<$ element name $=$ "statements.documetInformation"type $=$ "string" $>$

$<$ annotation $>$

$<$ documentation $>$ Section which contains information which describes the document $</$ documentation $>$

$<$ appinfo $>$

$<$ xbrl: rollup to $=$ "statements" weight $=$ "0" order $=" 1 " />$

$<$ xbrl:label xml:lang="en" $>$ Document Information $</$ xbrl:label $>$

$<$ appinfo $>$

$<$ annotation $>$

$</$ element $>$

$<$ element name $=$ "statements.balanceSheet"type $=$ "xbrl:monetary" $>$

$<$ annotation $>$

$<$ documentation $>$ Balance sheet issued by the entity $</$ documentation $>$

$<$ appinfo $>$

$<$ xbrl:rolluup to $=$ "statements"weight $=$ "0" order $=$ "4"/>

$<$ xbrl:label xml:lang="en" $>$ Balance Sheet $</$ xbrl:label $>$

$</$ appinfo $>$

$<$ annotation $>$

$</$ element $>$

$</$ schema $>$

Fonte: Vasal e Srivastava (2002, p.26). 


\section{Instance Document}

Neste documento se representam os fatos financeiros reais acontecidos na entidade no período relatado, expressos em termos de esquema XML, utilizando-se a taxonomia XBRL previamente definida. Nele são informados os valores assumidos pelas variáveis que já foram previamente definidas na taxonomia, no período considerado. $\mathrm{O}$ quadro 6 , a seguir, contém um exemplo de instance document de uma determinada entidade.

\section{QUADRO 6}

\section{Instance Document}

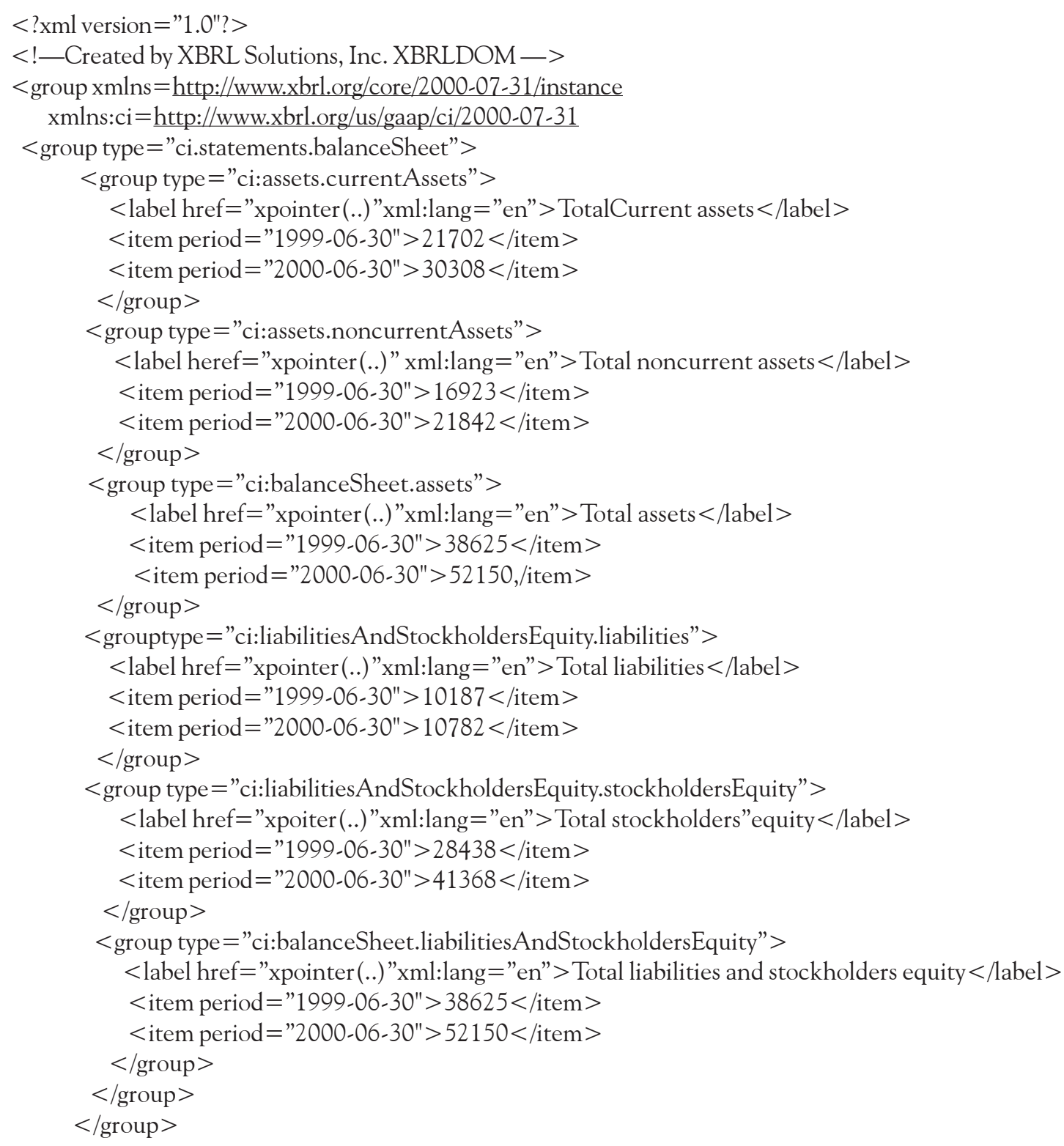

Fonte: Vasal e Srivastava (2002, p.28). 
O quadro 7 apresenta o balanço da entidade que serviu de exemplo, no caso a Microsoft, gerado em XBRL, utilizando-se a taxonomia do quadro 5 e o instance document do quadro 6.

\section{Style Sheet}

Como mencionado nos itens anteriores, o terceiro elemento da linguagem XBRL é o style sheet, que visa a permitir que as informações armazenadas no formato XBRL sejam disponibilizadas em qualquer formato desejado, ou criar inúmeros arquivos, como outro arquivo XML, arquivo print file PDF, uma página da web HTML, ou qualquer outro formato de arquivo. Desta maneira, um style sheet é composto de comandos que contêm informações de como será a saída dos dados do documento XBRL. Segundo Swagerman e outros, (2004), isso implica considerável economia de tempo e esforço na coleta, formatação e consolidação dos dados e na geração de relatórios para diferentes aplicadores. Um documento style sheet é escrito no formato XSL (eXtensible Style Language). A relação mútua entre um instance document, uma taxonomia e um style sheet é ilustrada na figura 2.

A figura mostra determinada taxonomia, previamente criada, sendo acessada para fornecer elementos que permitem validar os dados contidos no instance document, sendo que o style sheet é posteriormente acessado, a fim de se transformarem os dados, então no formato XBRL, para qualquer outro formato necessário, dependendo dos interesses e necessidades de seus usuários. O quadro 8, a seguir, apresenta um exemplo de um documento style sheet.

O quadro 9, a seguir, apresenta a respectiva saída obtida a partir da aplicação deste style sheet.

\section{FIGURA 2}

\section{QUADRO 7 \\ Balanço Condensado da Microsoft}

\begin{tabular}{lrr}
\hline Microsoft Corporation & Balance Sheets & In millions) \\
\hline June 30 & 1999 & 2000 \\
Assets & & \\
$\quad$ Total current assets & 21,702 & 30,308 \\
$\quad$ Total noncurrent assets1 & 6,923 & 21,842 \\
$\quad$ Total assets & $\$ 38,625$ & $\$ 52,150$ \\
Liabilities and stockholders" equity & & \\
$\quad$ Total liabilities & 10,187 & 10,782 \\
Total stockholders"equity & 28,438 & 41,368 \\
Total liabilities and stockholders'equity & $\$ 38,625$ & $\$ 52,150$ \\
\hline
\end{tabular}

Fonte: Vasal e Srivastava (2002, p.28).

Relacionamento entre os componentes do XBRL

Fonte: Swagerman e outros $(2004$, p.5).

\section{Relatórios Financeiros usando XBRL}

No Brasil, as companhias abertas devem obrigatoriamente divulgar suas demonstrações financeiras seguindo as diretrizes da Lei 6.404/76 (Lei das S.As) e também as instruções normativas da Comissão de Valores Mobiliários (CVM). Estas demonstrações financeiras representam o conjunto das informações referentes aos recursos e às obrigações dessas entidades, aos acionistas e a outros investidores, em determinado intervalo de tempo. Desta maneira devem ser apresentados o BP (Balanço Patrimonial), a DLPA (Demonstração de Lucros ou Prejuízos Acumulados), a DRE (Demonstração do Resultado do Exercício) e a DOAR (Demonstração das Origens e Aplicação dos Recursos), além das notas explicativas que complementam tais relatórios. 
Essas informações são de interesse de credores, investidores, analistas, entidades reguladoras, governo, associações, entre outros. Para Farewell (2004, p.2), cada um desses grupos requer as informações em um formato específico, e com isso as empresas devem buscar as informações em seus sistemas, transcrever nos formatos requeridos e submeter a cada grupo de usuários, que por sua vez transporta essas informações para seu sistema. O processo pode ser visualizado na figura 3, a seguir.

A XBRL, por sua vez, elimina a necessidade de transcrição de dados entre as aplicações, porque o dado passa a ser independente do aplicativo no qual ele é criado (os dados são mantidos de forma independente e sob uma denominação estabelecida e padronizada). Essa definição faz com que múltiplos usuários sejam capazes de extrair e reposicionar as informações financeiras. Assim, o próprio usuário recupera as informações financeiras pela Internet e passa para o formato que desejar, de acordo com suas necessidades. Este processo pode ser visualizado na figura 4 , a seguir.

\section{Aspectos sobre o uso do XBRL}

Alguns aspectos devem ser discutidos quanto à implementação da XBRL. A divulgação de informações financeiras das entidades sempre foi limitada ao material impresso, sendo que uma mudança para meio eletrônico certamente demandará estudos e discussões amplos, até que sejam aceitos e consolidados os novos procedimentos. Isto porque nela encontraremos vantagens e desvantagens nem sempre fáceis de serem avaliadas, muitas vezes compreendendo valores difíceis de serem mensurados, como agilidade, segurança, transparência e também os custos, difíceis de serem comparados, em parte devido à dificuldade de avaliar monetariamente benefícios intangíveis.

Costa e Silva (2004, p.2), em trabalho para a Universidade do Porto, Portugal, ao abordar o tratamento de informações, afirma que o modo como a informação é trabalhada atualmente não permite grande velocidade no tratamento dos dados, devido às diversas atividades que as entidades precisam executar, como preencher questionários ou alimentar bases de dados manualmente. Afirma ainda:

\section{QUADRO 8}

$<$ ?xml version $=" 1.0 " ?>$

<xsl:stylesheet xmlns:xsl=http://www.w3.org/1999/XSL/Transformversion="1.0">

(x) match $="$

$<$ TABLE BORDER $=" 1 "$

$<\mathrm{TH}>$ Part name $<\mathrm{TH}>$

$<\mathrm{TH}>$ Cost $</ \mathrm{TH}>$

$<\mathrm{TH}>$ Inventory at $\operatorname{Cost}</ \mathrm{TH}>$

$</ \mathrm{TR}>$

$<\mathrm{TR}>$

$<\mathrm{TD}><$ xsl:value-of select $=$ " partname" $/></ \mathrm{TD}>$

$<\mathrm{TD}><$ xsl:value-of select $=$ "cost $* \mathrm{QOH} " /></ \mathrm{TD}>$

$</ \mathrm{TR}>$

$</$ TABLE $>$

$</$ HTML $>$

template $>$

Fonte: Farewell (2003, p. 12).

\section{QUADRO9}

Dados XML formatados pela Style Sheet do Quadro 8

\begin{tabular}{l|r|r|r|r}
\hline $\begin{array}{l}\text { Part } \\
\text { Name }\end{array}$ & Cost & $\begin{array}{r}\text { Retail } \\
\text { Price }\end{array}$ & $\begin{array}{r}\text { Inventory } \\
\text { at Cost }\end{array}$ & $\begin{array}{r}\text { Inventory } \\
\text { at Retail }\end{array}$ \\
\hline Bolt & .59 & .99 & 44,25 & 74.25 \\
\hline Nut & .21 & .50 & 25.2 & 60 \\
\hline Screw & .15 & .24 & 14.25 & 22.8 \\
\hline Gear & 5.25 & 7.99 & 178.5 & 271,66 \\
\hline
\end{tabular}

Fonte: Farewell (2003, p. 13).

(...) havendo um método de extrair essa informação automaticamente, os agentes econômicos poderão analisar mais empresas, com os recursos que têm à sua disposição. Isto terá diversas conseqüências positivas: melhorará a representatividade das amostras utilizadas na base de diversas análises; aumentará a probabilidade de se extrair conhecimento relevante dos dados; diminuirá o erro esperado na inferência, a partir dos dados, de descritores do universo de referência. Assim sendo, 
as decisões dos agentes econômicos estarão mais bem fundamentadas e as pessoas que, nas diversas entidades, preenchem manualmente o formato da base de dados poderão dedicar-se a atividades de maior valor acrescentado, para si e para a entidade em que trabalham (COSTA E SILVA, 2004, p.2).

Pensando nisso, sente-se a necessidade de aproveitamento das potencialidades já existentes, de forma que os dados disponibilizados possam ser acessados de maneira automática, sem necessidade de redigitação pelos demais usuários. Essa idéia vem exatamente ao encontro dos potenciais recursos da XBRL. Sua integração, dentro de um sistema de informações, pode ser visualizada na figura 5, a seguir. Nela, vê-se o XBRL acessando o banco de dados da entidade, usar a taxonomia necessária para a formatação dos dados baseada nos princípios fundamentais de contabilidade e outras definições específicas para os usuários, permitir a entrada de dados e notas e gerar um arquivo no formato XML, que pode ser utilizado para as mais diversas finalidades, como os das agências regulatórias, impressão de demonstrações financeiras, geração de outros arquivos no formato de dados World ou Excell, por exemplo, e emitir as informações diretamente no meio Internet.

Para Silva e Teixeira (2004, p.26),

(...) o XBRL poderá ser o elo que falta no relato financeiro, para que a distribuição de informação financeira seja possível de forma fácil, rápida e eficiente, permitindo:

- reduzir o tempo e o custo de acesso e de preparação;

- facilitar o acesso e a distribuição;

- utilizar software de agentes inteligentes;

- adotar diferentes políticas contábeis, designadamente, modelos valorimétricos alternativos;

- relatar a informação utilizando taxonomias desenvolvidas especificamente para diferentes quadros alternativos;

- relatar informação de caráter dinâmico;

- aumentar e melhorar a análise da informação financeira e

- criar relatórios personalizados.
FIGURA 3

Informações financeiras sem o uso do XBRL

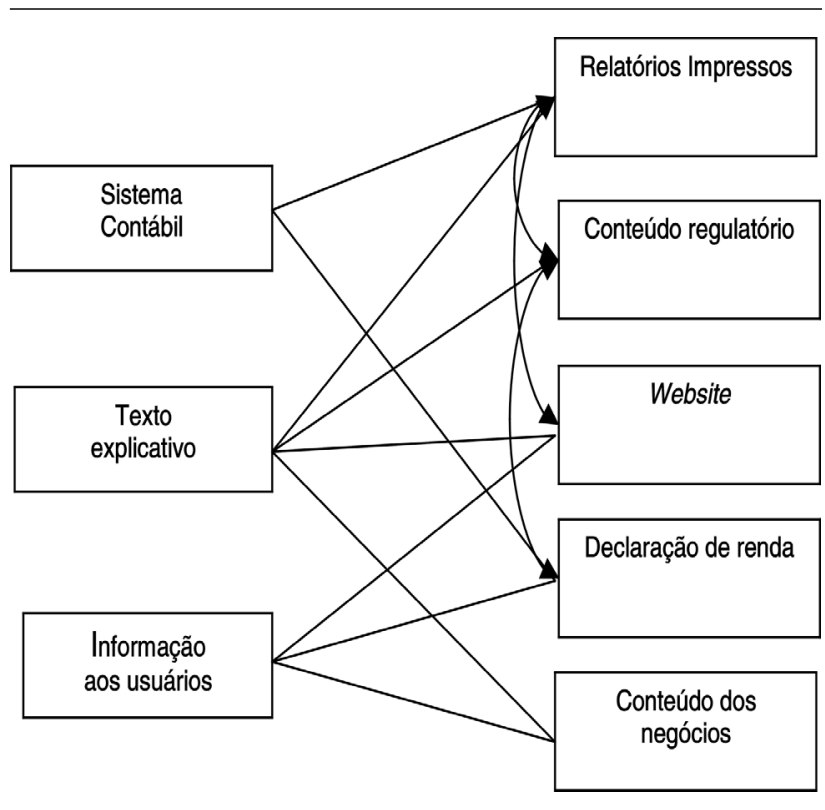

Fonte: Farewell (2003, p.3).

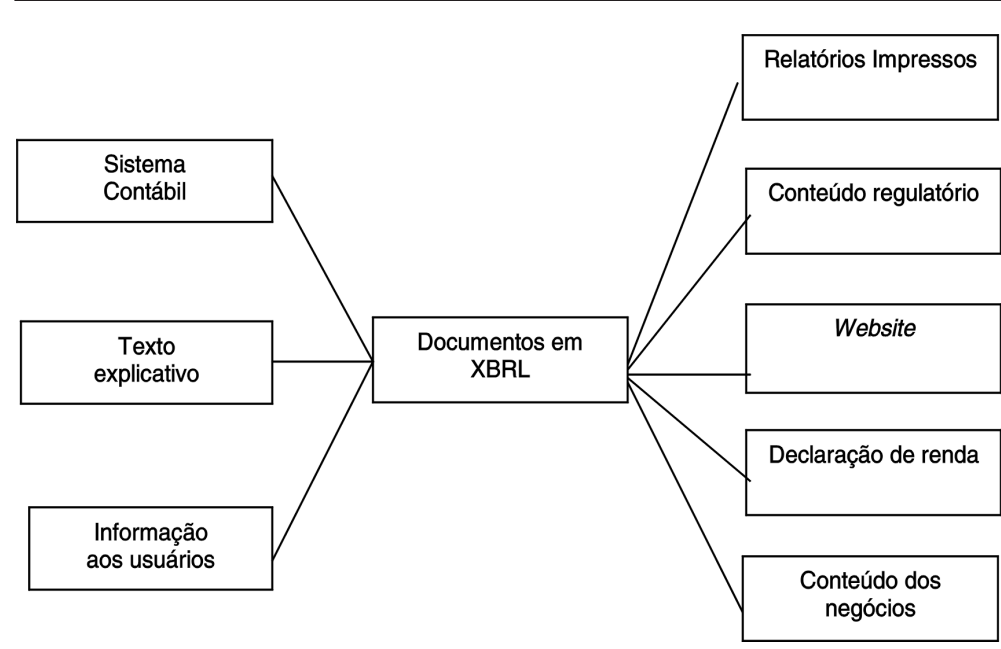

Fonte: Farewell (2003, p.4).
A utilização da XBRL deverá facilitar o agrupamento e a análise das informações, bem como a sua comparação. Também beneficiará a preparação de relatórios e sua análise. Sua utilização diminuirá os custos de preparação de relatórios e a freqüência de erros, uma vez que ela elimina a necessidade de redigitação de dados de um formato de relatório para outro. Para Silva (2003, p.26), 
a XBRL oferece vários benefícios, como independência de tecnologia, interoperabilidade, preparação eficiente de relatórios financeiros e facilidade de extração dos dados.

Além disso, se todos passarem a utilizá-la, ter-se-á uma padronização de informações que, em princípio, beneficiaria a todos, pois isso facilitaria o intercâmbio de informações entre as entidades e reduziria a necessidade de conversão freqüente de arquivos que ocorre atualmente. Haveria também maior divulgação das informações, pois se imagina que a Internet irá possibilitar acesso a um número significativamente maior de usuários interessados.

Para DiPiazza e Eccles (2004, p.3), o futuro do corporate reporting move-se inevitavelmente em direção a maior transparência e é preciso criar um novo ambiente de confiança, após as recentes revelações de escândalos financeiros. Afirmam ainda que:

XBRL vai desempenhar um papel essencial na obtenção de transparência empresarial e vai aumentar exponencialmente a velocidade com que os investidores podem obter e analisar a informação. Os investidores poderão solicitar informação a partir do seu software analítico e, em apenas alguns segundos, os dados de que necessitam serão incorporados na sua análise. Ao reduzir o tempo e os custos da reunião de dados, o XBRL também vai ajudar a nivelar o terreno de jogo para todos os investidores e outras partes interessadas.

Outra vantagem observada por Silva (2003, p.30) é a possibilidade de análises automatizadas e atualizações mais freqüentes da informação. Isso porque, com a padronização no fornecimento das informações, softwares especialistas serão desenvolvidos visando a depurar e avaliar as informações de maneira automatizada, reduzindo significativamente o tempo de análise e conseqüentemente os custos de recursos humanos necessários. E ainda, por ser um padrão aberto, o XBRL poderá ser adaptado por qualquer grupo às suas conveniências e necessidades, sem ter de arcar com um custo de utilização de software.

Alles e outros (2004, p.4), quando abordam os resultados da Lei Sarbanes-Oxley, editada após os recentes escândalos financeiros mundiais, visando a evitar ou, pelo menos, dificultar a ocorrência destes tipos de distorções na divulgação das informações, relatam:

A tecnologia de computação, tais como os sistemas de planejamento de recursos empresariais (ERP) e o surgimento do XML, especialmente o XBRL e derivativos do XBRL-GL, provê as firmas de maneira crescente da possibilidade de sistemas on-line, em tempo real de baixo custo. Isso poderia incluir demonstrativos financeiros atualizados até a última transação registrada... Isso cumpre os requisitos da Seção 409 da referida lei muito melhor que os passos preliminares tomados até então pela Comissão de Valores Mobiliários americana (SEC)... A premissa é de que corporações com sistemas de monitoramento e controles on-line terão menos latência (demora) nos seus processos e, conseqüentemente, irão ganhar vantagem competitiva sobre os outros atores nos seus setores (ALLES e outros, 2004, p.4).

E os aspectos negativos da linguagem, quais seriam? A primeira desvantagem a ser considerada são os custos de implementação iniciais, uma vez que ainda se necessita de muitos investimentos em materiais e recursos humanos, para que cada grupo possa desenvolver a(s) taxonomia(s) necessária(s) à sua área de atuação. A solução para pelo menos minimizar esses custos será a união das entidades comuns, canalizando recursos voltados para cada área, concentrando esforços e evitando duplicação de estudos e conseqüentemente de custos. Dessa forma, cada grupo vai determinar quais fatos 
financeiros necessita e deseja disponibilizar, criar a hierarquia entre esses fatos, expressar os relacionamentos e finalmente criar a taxonomia própria do segmento.

Outro aspecto a ser discutido é a questão da segurança. Ela envolve alguns riscos, como o de a informação transmitida não ser a mesma que chegue aos usuários, seja pela atuação de hackers ou pelo fato de a informação poder vir a ser disponibilizada parcialmente, ou mesmo quando se disponibilizam muitas informações, e não se tem claro qual delas foi realmente auditada e certificada. Roberto (2002, p.17) enumera algumas questões que precisam ser resolvidas quando se disponibilizam informações pela Internet:

1. identificar claramente qual informação é auditada e qual não é;

2. verificar se a informação está de fato segura;

3. verificar se o conjunto da informação não está prejudicado quando se publicam informações parciais;

4. certificar se a informação que chega é exatamente aquela que o auditor assinara;

5. certificar-se quanto à integridade das informações financeiras publicadas.

Em paralelo a isso, a fim de garantir a autenticidade e confiabilidade das informações eletrônicas, setores específicos do governo brasileiro estão começando a trabalhar as medidas visando a dar os primeiros passos para a implantação da chamada Certificação Digital das Informações. Isto está sendo discutido e considerado grande mudança para as companhias, que, junto a essa certificação, precisarão montar toda uma política de segurança das informações. Conforme Gonzaga (2004, p.1), "o Certificado Digital é um documento criptografado, que contém informações necessárias para identificação de uma pessoa física ou entidade jurídica”. Este certificado deverá ser emitido por uma autoridade competente autorizada pelo governo.

Resumindo os aspectos até aqui discutidos, elaboramos os quadros 10 e 11, respectivamente com as vantagens e desvantagens desta forma de divulgação.

A decisão então não deve se limitar apenas ao fator custo, mas principalmente aos benefícios que isto implicaria para a entidade, para o mercado financeiro e para a economia como um todo.

\section{QUADRO 10}

Vantagens na divulgação pela Internet, via XBRL

Padronização no formato dos dados
Facilidade de intercâmbio de informações
Eliminação de conversão freqüente de arquivos
Maior transparência em virtude da divulgação pela Internet e
maior penetrabilidade do meio
Facilidade de recuperação e utilização dos dados
Maior confiabilidade proporcionada aos analistas da informação
Possibilidade de integração na cadeia de suprimentos
Eliminação da redundância no fornecimento dos dados
Redução de erros
1

QUADRO 11

\section{Desvantagens na divulgação pela Internet, via XBRL}

\begin{tabular}{l|l}
\hline 1 & Custos de implementação iniciais \\
2 & Custos de manutenção constante de web sites \\
3 & $\begin{array}{l}\text { Necessidade de certificação constante da integridade da } \\
\text { informação disponibilizada }\end{array}$ \\
4 & $\begin{array}{l}\text { Custo da certificação digital } \\
\text { Possibilidade de se receber simultaneamente informações } \\
\text { auditadase não auditadas }\end{array}$ \\
6 & $\begin{array}{l}\text { Possibilidade de ocorrer publicação de informações parciais } \\
\text { que podem prejudicar o conjunto da imagem das entidades }\end{array}$ \\
\hline
\end{tabular}

\section{O Consórcio XBRL Internacional}

O Consórcio XBRL International é sediado nos Estados Unidos e reúne atualmente cerca de 250 companhias do mundo, entre entidades públicas, privadas, associações e agências governamentais. Este consórcio regula as chamadas Jurisdições presentes em vários outros países do mundo, objetivando dedicar estudos, desenvolver e incentivar o desenvolvimento e manutenção das taxonomias para adoção do XBRL no país. Cada jurisdição é a representação de um país, uma região ou um regime internacionalmente reconhecido de regulação de normas contábeis ou financeiras, sendo que, pelas regras do consórcio, existem dois tipos de jurisdições, as estabelecidas e aquelas ainda provisórias, que têm até dois anos para se tornarem estabelecidas. Existe ainda a categoria direct participants, que serve a organizações localizadas em países em que ainda não há jurisdição formada, mas que estão interessadas em participar desses estudos (XBRL INTERNATIONAL, 2004).

Atualmente, dez países no mundo já têm jurisdição estabelecida: Alemanha, Austrália, Canadá, Espanha, Estados Unidos, Holanda, Inglaterra, Irlanda, Japão, Nova 
Zelândia. Além desses, também já consta como uma jurisdição o IASB, por sua representatividade em âmbito mundial. Contam com jurisdição provisória a Bélgica, a Coréia do Sul, a Dinamarca, a França e a Suécia. Existem ainda outros países que não formalizaram uma jurisdição, mas já iniciaram esse processo. São eles a Áustria, China, Eslovênia, Finlândia, Grécia, Hungria, Itália, Luxemburgo, Noruega, Portugal, República Tcheca, Singapura e Suíça.

\section{Eventos específicos abordando o XBRL}

A XBRL Internacional e a IASC Foundation, desde o ano de 2001, promovem, duas vezes por ano, conferências internacionais de XBRL, nas quais são anunciados novos produtos, projetos e desenvolvimentos, vários deles envolvendo o XBRL. A última edição, a $11^{\text {th }}$ XBRL International Conference foi realizada de 25 a 28 de abril de 2005 em Boston, Massachusetts, EUA, sendo que a próxima dessas conferências, que será a $12^{\text {th }}$ XBRL International Conference, está agendada para acontecer de 07 a 10 de novembro de 2005 e deverá ser realizada em Tokyo, Japão.

E no Bryant College, na cidade de Smithfield, Rhode Island, USA, um centro de pesquisas na tecnologia XBRL, realiza-se anualmente a chamada XBRL Conference at Bryant College, que trata dos temas taxomomia, desenvolvimento de aplicações e pesquisas em XBRL (BRYANT, 2004).

E também, nestes países onde os estudos já se iniciaram, realizam-se conferências, congressos, reuniões e workshops tratando do tema, como, por exemplo, o que se anuncia para acontecer em Bogotá, Colômbia, em 13

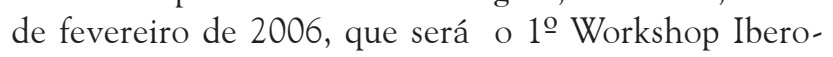
americano de XBRL, que visa a discutir o assunto e integrar a comunidade XBRL Ibero-americana (XBRL COLOMBIA, 2005).

\section{A difusão do XBRL no Brasil}

No Brasil já despontam estudos e trabalhos acadêmicos envolvendo o tema XBRL, como dissertações defendidas na Universidade de São Paulo e Universidade Federal da Bahia. $\mathrm{Na}$ área governamental, mais especificamente no Banco Central do Brasil, Silva (2003) identificou três ambientes de informações financeiras que julga comuns a qualquer órgão público, identificou os benefícios que o XBRL poderia trazer ao setor, propôs o desenvolvimento de taxonomias para as demonstrações financeiras do Banco Central e para aquelas que lhe são repassadas pelas entidades financeiras, além de já haver desenvolvido uma proposta de taxonomia para representação dos balanços patrimoniais daquele banco governamental, utilizando XBRL. Em 2004 e 2005, realizaram-se o 1ํe o 2을 Contecsi - Congresso Internacional de Gestão de Tecnologia e Sistemas de Informação TECSI/FEA/USP, Laboratório de Tecnologia e Sistemas de Informação da FEA/USP, em que se discutiu o assunto XBRL, visando a uma abordagem de experiências na utilização da linguagem (TECSI, 2005).

O 1ํㅡorkshop Brasileiro de XBRL, coordenado pelo professor doutor Edson Luiz Riccio, da FEA USP, contou com representantes da USP e de outras universidades, membros de instituições governamentais e representativas de classe, como o Banco Central do Brasil, Comissão de Valores Mobiliários, Bovespa, Fundação Brasileira de Contabilidade, CFC (Conselho Federal de Contabilidade), Ibracon (Instituto dos Auditores Independentes do Brasil), Animec (Associação Nacional de Investidores do Mercado de Capitais, Sindcont-SP, além de cerca de 40 instituições privadas e líderes nos estudos do XBRL (WORKSHOP1 XBRL, 2004).

Por último, em 5 de outubro de 2005, foi realizado, pelo TECSI FEA USP em São Paulo, o $2^{\mathrm{o}}$ Workshop Brasileiro de XBRL, que contou mais uma vez com representantes de diversas entidades públicas e privadas nacionais e teve como palestrantes internacionais convidados a diretora de XBRL at Edgar On-line Inc, entidade que promove a divulgação de informações financeiras das empresas participantes da Nasdaq, entidade equivalente à nossa Bovespa, e ainda, via Internet, diretamente dos Estados Unidos (WORKSHOP2 XBRL, 2005).

\section{CONSIDERAÇÕES FINAIS E RECOMENDAÇÕES}

A Internet provocou uma revolução no mundo, nas formas de comunicação e troca de informações. A linguagem XBRL, uma das formas da linguagem XML, foi e está sendo desenvolvida no mundo para facilitar a divulgação de demonstrativos financeiros por meio desse novo meio de comunicação.

Para adaptar a linguagem XBRL aos demonstrativos financeiros de cada país, são necessários estudos visando a adaptar a linguagem ao tipo de demonstrativo financeiro divulgado em cada setor de cada um desses países, de acordo com os princípios fundamentais de contabilidade neles adotados. Essa adaptação é a construção da taxonomia voltada para os seus demonstrativos financeiros, seus formatos e suas regras. Esse é o desafio que cada setor de cada um desses países 
está encarando para construir suas próprias taxonomias. Depois de montadas essas taxonomias, as informações poderão ser divulgadas com muito mais dinamismo, agilidade e flexibilidade, e a troca de informações entre diferentes entidades e mesmo entre diferentes países será possível sem que se necessite de trabalhosas e desgastantes conversões nos formatos das informações.

Pelas características observadas da linguagem e pelo desenvolvimento já observado em vários países do mundo, podemos concluir que a linguagem XBRL tende a ser e deve vir a ser padronizada para divulgação de informações financeiras pela Internet. Quanto às mudanças de padrão, com a adoção mundial do XBRL, os diversos padrões de divulgação das informações financeiras deverão ser unificados em um único formato, de acordo com a taxonomia adotada, com isto visando a gerar maior interatividade, acessibilidade, extensibilidade (criação de novos elementos), confiabilidade e conectividade das informações. Em diversos países, grupos compostos por acadêmicos, membros de órgãos governamentais e desenvolvedores de softwares estão sendo formados a fim de estudar maneiras de se adaptarem às necessidades geradas pela nova forma de apresentação de informações financeiras.

No Brasil, a opção pelo mercado globalizado e a intenção de participar de mercados comuns dos Estados Unidos e Europa devem fazer o país alinhar-se às iniciativas já adotadas por outros países, para a padronização da divulgação de informações financeiras. Os estudos apontam para a plena utilização da linguagem XBRL. As ações desenvolvidas por grupos acadêmicos liderados pelo TECSI/FEA/USP e o sucesso verificado em seus três workshops brasileiros de XBRL, além de reuniões com representantes de importantes entidades governamentais, apontam para um crescimento dos esforços nesses estudos e pesquisas e a introdução efetiva do XBRL no Brasil.

O caminho para a implantação do XBRL em nosso país aponta ser aquele seguido pelos demais países que já iniciaram esse processo, que é o de formar um grupo de pesquisas, sediado por uma entidade governamental ou reguladora do mercado financeiro ou contábil, como CVM, Banco Central, CFC, Bovespa, Receita Federal e outros, e concentrar esforços na criação das taxonomias necessárias para os diversos tipos de usuários.

Em nosso país, os estudos foram iniciados, existindo ainda um amplo campo de pesquisas, dentre os quais se pode recomendar:
- desenvolver taxonomias brasileiras para relatórios contábil-financeiros, obedecendo aos Princípios Fundamentais de Contabilidade Brasileiros, cada uma aplicada às diversas áreas de atuação das entidades, como comercial, industrial, bancária, setor público, terceiro setor etc. (O TECSI FEA USP está liderando este desenvolvimento desde outubro de 2003, apoiado pelo XBRL Institute dos Estados Unidos);

- desenvolver outras taxonomias voltadas para a divulgação de informações gerenciais e para usuários internos às empresas;

- elaborar estudos comparativos dos custos em se continuar a divulgação no formato atual utilizado, e aqueles que se teriam, passando-se ao padrão XBRL, tentando-se inclusive medir valores intangíveis alcançados com a utilização da linguagem;

- elaborar protótipos de divulgação de informações financeiras, visando a estudar o desempenho do XBRL, quando comparado com outras formas de divulgação.

- desenvolver taxonomias destinadas a comunidades específicas dentro do país, tais como para o setor elétrico, de seguros e saúde, tribunais de contas, a União, estados e municípios, entre outros, onde o fluxo de informações é complexo e de grandes volumes.

É certo que a implementação da linguagem XBRL iniciarse-á pela área contábil-financeira, voltada às empresas de capital aberto, que estão sujeitas ao controle pelas agências reguladoras e pelo mercado. Também são as que se enquadram nas regras nacionais e internacionais de governança corporativa e de divulgação de informações, como as constantes do Novo Mercado, criado pela Bovespa.

No entanto, é de se esperar que todas as empresas, mesmo as de capital fechado e as empresas públicas, divulguem suas informações aos órgãos reguladores e ao público utilizando a linguagem XBRL.

O processo de introdução do XBRL nos diversos países tem variado, como se percebe neste trabalho. No entanto, as características de cada país influenciam o processo. Por ser o Brasil um dos muitos países onde existe a tradição do chamado "Code Law", é natural que as empresas só adotem certas práticas quando isso se torna lei ou quando existe forte orientação dos órgãos reguladores sinalizando às empresas que o caminho será esse. 
Note-se, como exemplo, que a Securities Exchange Commision (SEC), órgão regulador do mercado de ações norte-americano, equivalente à Comissão de Valores Mobiliários brasileira, estabeleceu, em 3 de feverreiro de 2005, um programa para que as empresas que negociam suas ações no mercado norte-americano, a Bolsa de Nova Iorque, sejam voluntárias ao uso do XBRL na divulgação de suas informações contábil-financeiras. Esse programa, chamado de Voluntary Filing Program, já contava, em outubro de 2006, com 20 empresas de renome mundial. Entre essas, quatro são brasileiras: Banco Itaú, Petrobras, Gol e Net Serviços de Comunicação. Nesse caso, essas empresas já estão utilizando o XBRL norte-americano, pois têm de reportar seus dados para a SEC. Assim que a taxonomia XBRL brasileira seja criada e colocada em fase de testes e validada pelos órgãos reguladores, espera-se que os mesmos adotem medida semelhante à da SEC, ou seja, induzindo as empresas participantes da Bovespa a adotar a nova tecnologia, até que isso se torne compulsório por meio de lei específica.

Artigo submetido em 20/10/2005 e aceito em 13/03/2007.

\section{REFERÊNCIAS}

ALIANA, Toni. XBRL: el reporting financiero sobre Internet. Disponível em <http://www.asset.es/miembros/ articulo_antoni_aliena.doc $>$. Acesso em: 23 Nov. 2004.

ALLES, Michael et al. A lei das consequências não intencionais?: avaliando os custos, benefícios e resultados da lei Sarbanes-Oxley. São Paulo: ISACA, 2004. Disponível em: < http://www.isaca.org.br/>. Acesso em: 20 ago. 2004.

BRASIL. Lei 6.404 de 15 de dezembro de 1976. Dispõe sobre as sociedades por ações. Diário Oficial [da] República Federativa do Brasíl, Brasília, DF, 1976.

BRYANT UNIVERSITY. What is the history of XBRL?. Smithfield, 2004. Disponível em: <http://web.bryant.edu/ xbrl/xbrl/history.htm $>$. Acesso em 23 nov. 2004.

CHEMICAL MARKUP LANGUAGE - CML. Chemical Markup Language. 2005. Disponível em: < http://www.xml-cml.org/main.html>. Acesso em: 04 jan. 2005.

DEBRECENY, Roger; GRAY, Glen L. Are we there yet?: a reserch perspective on the extensible business reporting language (XBRL). Singapore: [s. n.], 2004.

DIPIAZZA, Sam; ECCLES, Robert G. Necessária reforma global do corporate reporting para restaurar a confiança pública nos mercados de capitais. Disponível em: < http://www.pwcglobal.com/pt/por/about/pressrm/info media/buildingpt.html/>. Acesso em: 08 jun. 2004.

EBXML. Electronic business information. Disponível em < $<$ http:// www.ebsml.org/>. Acesso em: 04 jan. 2005.
FAREWELL, Stephanie. A plan for implementing XBRL in an Introductory AIS Course. In: AAA-IS SECTION MID-YEAR CONFERENCE, 2004. Proceedings... [Arkansas]: University of Arkansas at Little Rock, 2004.

FINXML. The digital language for capital markets. 2005. Disponível em: $<$ http://www.finxml.org/>. Acesso em: 04 jan. 2005.

FPML. Financial products markup language. 2005. Disponível em $<$ http:/ 〈fpml.org/>. Acesso em: 04 jan. 2005.

GIDDENS, Anthony. (1999) Un mundo desbocado: los efectos de la globalización en nuestras vidas. Madri: Santillana, 2000.

GONZAGA, Diogo C. Certificação digital. 2004. Disponível em: < http:/ br.linux.org/tutoriais/002209.html/>. Acesso em >: 15 fev. 2005.

HOFFMAN, Charles; STRAND, Carolyn. XBRL essentials. New York: AICPA, 2001.

HR-XML CONSORTIUM. XML for human resource. 2005. Disponível em: <http://www.hr-xml-org/channels/home.htm/>. Acesso em: 04 jan. 2005.

INTERNATIONAL ORGANIZATION FOR STANDARDZATION - ISO. International Organization for Standardization. Disponível em: < http://www.iso.org >. Acesso em: 30 nov. 2004.

LIGHT, Richard. Iniciando em XML. São Paulo: Makron Books, 1999.

MERRIAN-WEBSTER Online dictionary. Disponível em: < $\underline{\text { http:// }}$ www.m-w.com/cgi-bin/dictionary?book $=$ Dictionary\&va $=$ taxonomy $>$. Acesso em: 29 maio 2004.

PICONEZ, Stela T. B. EDM5053: ambientes de aprendizagem cooperativa apoiados em tecnologias da internet: novos desafios, novas competências. São Paulo: FE/USP, 2003.

RAY, Erik. T. Aprendendo XML. Rio de Janeiro: Campus, 2001.

REVISTA DE GESTÃO DA TECNOLOGIA E SISTEMAS DE INFORMAÇÃO, v. 2, n. 3, 2005. 2o Workshop Brasileiro de XBRL. Disponível em:

$<$ http://www.tecsi.fea.us.br/revistatecsi/edicoesanteriores/v02n03. 2005/v02n03-2005/Relatorio2XBRL/relatorioXBRL2.html>. Acesso em: 07 out. 2005.

ROBERTO, José Gonçalves. Relato tradicional versus o relato numa forma electrónica. Revisores \& Empresas, Lisboa, ano 4, n. 17, p. 16 28, abr.jjun. 2002.

SILVA, Ana Costa e. Extracção de informação de tabelas contidas em texto: uma aplicação a relatórios e contas de empresas portuguesas. 2003. Dissertação (Mestrado em Análise de Dados e Sistemas de Apoio à Decisão) - Faculdade de Economia da Universidade do Porto, Porto, 2003.

SILVA, Paulo Caetano da. Explorando linguagens de marcação para representação de relatórios de informações financeiras. 2003. Dissertação (Mestrado Profissional em redes de computadores) - Programa de PósGraduação em Redes de Computadores da Universidade Salvador, Salvador, 2003.

; SULAIMAN, Alberto. XBRL, regras de negócios e relatórios financeiros. São Paulo: Kmbrasil, 2003.

; TEIXEIRA, Cesar Camilo. A gestão da informação financeira do Banco Central do Brasil apoiada por XBRL. Salvador: [s. n.], 2004. 
SWAGERMAN, D.M. et al. Application of XBRL for local authorities. Twente: [s. n.], 2004.

TECSI. Laboratório de Tecnologia e Sistemas de Informação. In: CONGRESSO INTERNACIONAL DE GESTÃO DE TECNOLOGIA E SISTEMAS DE INFORMAÇÃO. Anais eletrônicos... Disponível em: <http://www.tecsi.fea.usp.br/eventos/contecsi2004>. Acesso em: 31 jul. 2004.

TOP. TOP 10 Technologies confirms interest in information security, spam control. The CPA Journal, New York, v. 74, n. 4, p. 15, Apr. 2004. Disponível em: < http://www.nysscpa.org/cpajournal/2004/404/ perspectives/nv11.htm >. Acesso em: 20 set. 2005.

TRUMAN STATE UNIVERSITY. A presentation on XBRL. Disponível em: < http://www2.truman.ed/ a1690/xbrl2.htm>. Acesso em: 23 nov. 2004.

VASAL, Virendra K.; SRIVASTAVA, Rajendra P. Extensible Business Reporting Language (XBRL): the digital language of business: an Indian perspective. Indian Accounting Review, v. 6, n. 1, p. 41-59, June 2002.

XBRL HISTORY. XBRL history. New York, 2004. Disponível em: < http:/ /www.xbrl.org/history-print.aspx>. Acesso em: 23 nov. 2004.

XBRL INTERNATIONAL. Progress report: Abril 2004. New York, 2004. Disponível em: < http://www.xbrl.org/>. Acesso em: 23 nov. 2004.
XBRL (Irlanda). About XBRL Ireland. 2005. Disponível em: < http:// www.xbrl-ie.org/public/aboutus.htm/>. Acesso em: 05 set. 2005.

XBRL 1O. Top findings of the $10^{\text {th }}$ XBRL International Conference in Brussels. Belgium, 2005. Disponível: <http://www.xbrl.org// brussels\%20Top\%20Findings_Final.htm/>. Acesso em: 18 fev. 2005.

XMLNEWS.ORG. XML and the news industry. 2005. Disponível em: $<$ http://www.xmlnews.org/>. Acesso em: 04 jan. 2005.

WEBOPEDIA: first online encyclopedia dedicated to computer technology. 2004 Disponível em: < http://www.webopedia.com $>$. Acesso em 30 nov. 2004.

WORKSHOP BRASILEIRO DE XBRL, 1., 2004. Anais eletrônicos...Disponível em: <http://www.tecsi.fea.usp.br/eventos/ workshopxbrl01>. Acesso em 31 jul. 2004.

WORKSHOP IBEROAMERICANO DE XBRL, 1., Bogotá. Anales elctrónicos... Bogotá: [s. n.], 2005. Disponível em: < http:// integraxml.com/workshop2006/>. Acesso em: 19 set. 2005.

ZAROWIN, Stanley; HARDING, Wayne E. Finally, business talks the same language. Journal of Accountancy Online Issues, Aug. 2000. Disponível em: < http://www.aicpa.org/pubs/jofa/aug2000/ zarowin.htm $>$. Acesso em: 23 nov. 2004. 